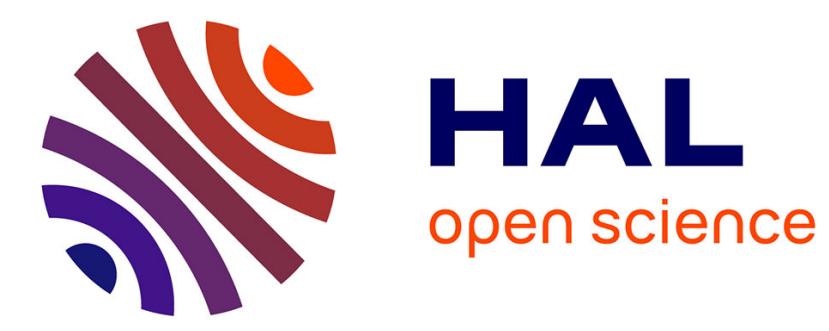

\title{
Measurements of High Density Plasmas after Pulsed Discharges in Liquid Helium
}

\author{
W. Qin, K. Minami, A. Desilva, F. Tomimoto
}

\section{To cite this version:}

W. Qin, K. Minami, A. Desilva, F. Tomimoto. Measurements of High Density Plasmas after Pulsed Discharges in Liquid Helium. Journal de Physique IV Proceedings, 1997, 07 (C4), pp.C4-319-C4-329. 10.1051/jp4:1997426 . jpa-00255582

\section{HAL Id: jpa-00255582 https://hal.science/jpa-00255582}

Submitted on 1 Jan 1997

HAL is a multi-disciplinary open access archive for the deposit and dissemination of scientific research documents, whether they are published or not. The documents may come from teaching and research institutions in France or abroad, or from public or private research centers.
L'archive ouverte pluridisciplinaire HAL, est destinée au dépôt et à la diffusion de documents scientifiques de niveau recherche, publiés ou non, émanant des établissements d'enseignement et de recherche français ou étrangers, des laboratoires publics ou privés. 


\title{
Measurements of High Density Plasmas after Pulsed Discharges in Liquid Helium
}

\author{
W. Qin, K. Minami, A.W. DeSilva* and F. Tomimoto \\ Graduate School of Science and Technology, Niigata University, Niigata 950-21, Japan \\ * Institute for Plasma Research, University of Maryland, College Park, MD 20742, U.S.A.
}

\begin{abstract}
High density plasmas created by high-voltage pulsed discharges in liquid helium below $4.2 \mathrm{~K}, 1$ atm, and cryogenic helium gas near $4.2 \mathrm{~K}, 1 \mathrm{~atm}$, have been investigated by spectroscopic measurements in the visible spectral range. The well known atomic lines exhibit strong and broadened profiles with underlying continua at early stages. Several experimental findings indicate that this broadening is dominated by Stark broadening caused by the presence of the transient plasmas. The spectral profiles are therefore used to deduce the plasma densities, which are on the order of $10^{18} \mathrm{~cm}^{-3}$ at $0.2 \mu \mathrm{s}$ after the breakdown. The electron temperatures are measured from the ratios of total spectral line intensities to continuum intensities. It turns out to be about $35,000 \mathrm{~K}$ at $0.2 \mu \mathrm{s}$. The measured plasma parameters suggest that the observed plasmas are moderately strongly coupled plasmas with Coulomb coupling coefficients around 0.1. In addition, some strong spectral features are observed near $430 \mathrm{~nm}$, which can be assigned to $\mathrm{He}_{2}$ transition $\left(g^{3} \Sigma_{\mathrm{g}}^{+}-a^{3} \Sigma_{u}^{+}\right)$. The reaction mechanisms are discussed in this paper.
\end{abstract}

\section{INTRODUCTION}

For many years, a variety of high-density plasmas that were beyond the scope of conventional laboratory experiments have been generated by novel methods such as laser implosion of a small target [1, 2]. Azechi et al. [2] generated a strongly coupled (non-ideal) and partly degenerated plasma with density on the order of $10^{26} \mathrm{~cm}^{-3}$ and electron temperature of $0.5 \mathrm{keV}$ attained by the laser implosion. Here, the strongly coupled plasma means that the Coulomb coupling coefficient $\Gamma$ defined by the ratio of the average Coulomb energy to the average kinetic energy is not much less than unity. Shock wave compression in gases or solid materials have been measured to generate non-ideal plasmas with densities on the order of $10^{18} \mathrm{~cm}^{-3}$ and electron temperature around $1 \mathrm{eV} \mathrm{[3].} \mathrm{One} \mathrm{of} \mathrm{the} \mathrm{authors} \mathrm{(A.} \mathrm{W.} \mathrm{DeSilva)}$ and his collaborator measured by means of laser scattering a plasma with densities $10^{17} \mathrm{~cm}^{-3}$ and electron temperature $2 \mathrm{eV}$ [4]. Peaked spectra caused by ion-acoustic fluctuations in the collision dominated plasmas were detected and fitted to Bhatnagar-Gross-Krook model among various versions.

On the other hand, spectroscopic measurements have been employed extensively to investigate properties of dissolved impurities in liquid helium (LHe). Spectroscopic measurements were carried out to measure dissolved clusters from metal target caused by laser ablation [5]. Laser-generated sparks have been employed to analyze liquids spectroscopically [6]. Our spectroscopic study presented here was motivated by a possible existence of cryogenic plasma states generated by high-voltage pulsed discharges in LHe [7]. The cryogenic plasma, i.e., positive and negative ion mixture with temperature below $4.2 \mathrm{~K}$ dissolved in LHe, may be an unknown subject in physics of condensed matter. Timeresolved emission spectra resulting from sparks in LHe and cryogenic helium (He) gas are here measured in the visible spectral region from $380 \mathrm{~nm}$ to $720 \mathrm{~nm}$. In LHe, a high power discharge will create a gaseous He bubble between the electrodes, as occurred in other liquids [6]. The measurements we made, in fact, are limited to transient gaseous He plasmas in cryogenic environments immediately after the pulsed discharge. The results show that the well known neutral He atomic lines are strong and broadened with underlying continua at the early stages. Several experimental findings indicate that this broadening is dominated by Stark broadening caused by the presence of the transient plasmas. The 
spectral profiles are therefore used to deduce the plasma densities. The electron temperatures are measured from the ratios of total spectral that the observed plasma with relatively high density and low electron temperature due to rapid cooling are moderately strongly coupled plasmas beyond the scope of conventional discharges in gas at room temperature. In addition, there are some strong spectral features near $430 \mathrm{~nm}\left(23300 \mathrm{~cm}^{-1}\right)$, which can be assigned to the $\mathrm{He}_{2}$ transition $\left(g^{3} \Sigma_{g}{ }^{+}-a^{3} \Sigma_{u}{ }^{+}\right)$. They are not observed in $77 \mathrm{~K}$ and $300 \mathrm{~K}, 1 \mathrm{~atm}, \mathrm{He}$ gas under the same experimental conditions. By analyzing these spectral data, the emissions of neutral atomic $\mathrm{He}$ are estimated to be from the hot core of the plasma while the strong molecular $\mathrm{He}_{2}$ transitions occur at the rapid cooling edge layers, where the molecular He ions become dominant positive ions.

The outline of this paper is as follows: the experiment is described in section 2, analyses and discussions of the measured results are given in section 3 , and conclusions are presented in section 4 .

\section{EXPERIMENTS}

Pulsed discharges of $-22 \mathrm{kV}$ are made between tungsten rod electrodes separated by $1.5 \mathrm{~mm}$ in a Dewar bottle filled with LHe or with.cryogenic He gas. The peak discharge current measured by a Rogowski coil is about $50 \mathrm{~A}$ with $0.5 \mu \mathrm{s}$ duration as shown in Figure 1. The states of He examined are (1) superfluid LHe II with temperature $1.62 \mathrm{~K}$ and pressure $5.8 \mathrm{Torr}$ (2) normal fluid LHe with $4.2 \mathrm{~K}$ and $1 \mathrm{~atm}$, (3) cryogenic He gas near $4.2 \mathrm{~K}$ and $1 \mathrm{~atm}$, (4) He gas near $77 \mathrm{~K}$ and $1 \mathrm{~atm}$, and (5) He gas at $300 \mathrm{~K}, 1 \mathrm{~atm}$. The case (1) is attained by evacuation cooling, i.e., the pressure of He gas above the LHe surface is kept at 5.8 Torr. The cases (4) and (5) are investigated for comparison.

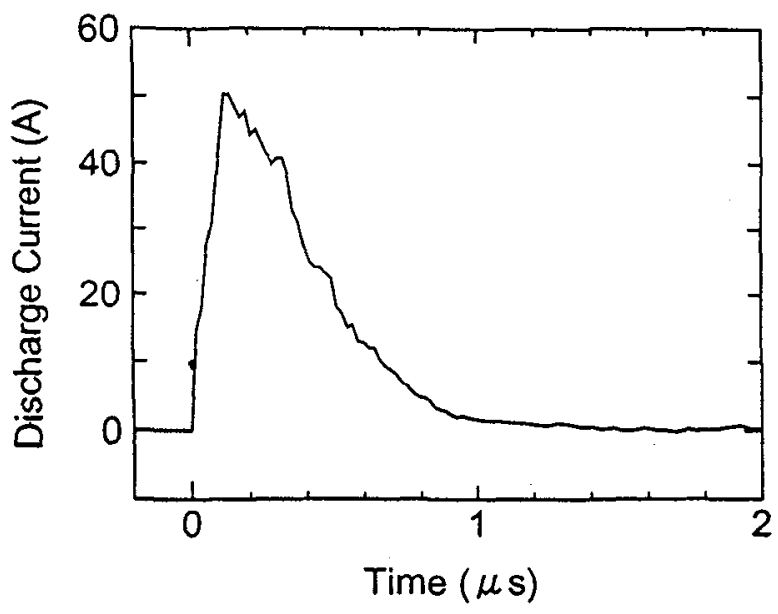

Figure 1: The discharge current vs time measured by a Rogowski coil.

The light emissions from the spark are collected through a vertical transparent slit made on the Dewar bottle by a lens and focused into the entrance slit of a $0.25 \mathrm{~m}$ monochromator fitted with a 1200 grooves/mm grating. The detector is a MCP high-speed gated image intensifier head combined with a multichannel detecting system. The 1024 channels cover a spectral region of $46 \mathrm{~nm}$ when the center wavelength of the monochromator is set to $550 \mathrm{~nm}$. Spectra at various delay times $t_{d}$ after the discharge can be measured in a certain spectral range without the necessity for scanning the spectrometer. The time resolution is $0.4 \mu \mathrm{s}$. No particular efforts have been made for getting spatial resolution. The data are average values of all line-of-sight emissions. The calibration of the spectral response is carried out by using a tungsten-filament $2,854 \mathrm{~K}$ standard lamp. 


\section{ANALYSES AND DISCUSSIONS}

\subsection{Determination of Plasma Densities}

\subsubsection{Spectral Line Broadening}

The emissions from discharges in all five cases (1)-(5) in a spectral region from $380 \mathrm{~nm}$ to $720 \mathrm{~nm}$ last typically about $6-8 \mu \mathrm{s}$. In the low temperature cases (1)-(3), the spectra show extremely broadened neutral He atomic lines together with a very strong continuum background at the early stage after the discharge. Then, the continua gradually disappear. Most of the strong atomic lines $388.9 \mathrm{~nm}\left(3 p^{3} \mathrm{P}\right.$ $\left.2 s^{3} \mathrm{~S}\right), 447.1 \mathrm{~nm}\left(4 d^{3} \mathrm{D}-2 p^{3} \mathrm{P}\right), 501.7 \mathrm{~nm}\left(3 p^{1} \mathrm{P}-2 s^{1} \mathrm{~S}\right), 587.6 \mathrm{~nm}\left(3 d^{3} \mathrm{D}-2 p^{3} \mathrm{P}\right), 667.8 \mathrm{~nm}\left(3 d^{1} \mathrm{D}-2 p^{1} \mathrm{P}\right)$ and $706.5 \mathrm{~nm}\left(3 s^{3} \mathrm{~S}-2 p^{3} \mathrm{P}\right)$ in this spectral region appear and become increasingly narrow in line width as time elapses. An example of case (1) near the spectral line $587.6 \mathrm{~nm}$ is shown in Figure 2.

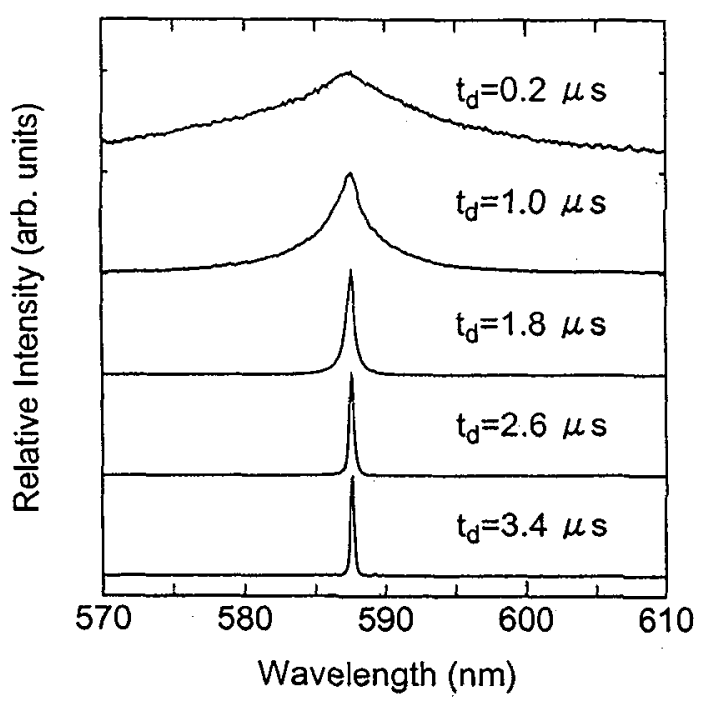

Figure 2: Time-resolved emission spectra near $587.6 \mathrm{~nm}\left(3 d^{3} \mathrm{D}-2 p^{3} \mathrm{P}\right)$ in $1.62 \mathrm{~K}, 5.8$ Torr liquid helium. Note the line broadening and continuum at early stage.

For the gas cases in our conditions, the observed spectral line broadening can be considered to be dominated by Stark broadening as in usual dense plasmas. The plasma densities can be accordingly calculated with the use of the theory developed by Griem and his co-workers [8-10]. In LHe cases (1) and (2), however, the plasma is believed to be located in the gas bubble bounded by LHe, in which the pressure may be very high and decreases rapidly as time progresses [6]. The line width, therefore, may result partially from the effects other than that of the local electrons and ions. Nevertheless, the following experimental findings indicate that the broadening in LHe is also dominated by Stark broadening.

Firstly, the measured spectral line profiles and numerically calculated Stark profiles can be well fitted with each other. The numerical calculations of the Stark spectral line profiles are based on the impact approximation for electrons and quasi-static approximation for ions as were described by Griem and the co-workers [8-10]. Two examples of the fittings for the spectral line $587.6 \mathrm{~nm}$ at delay time $0.2 \mu \mathrm{s}$ and $1.4 \mu \mathrm{s}$, respectively, in the case (1) are shown in Figure 3. The poor fit at the line center is probably due to contributions from low-density edge layers because of the spatial and temporal nonuniformity of plasma parameters in the discharge region. 


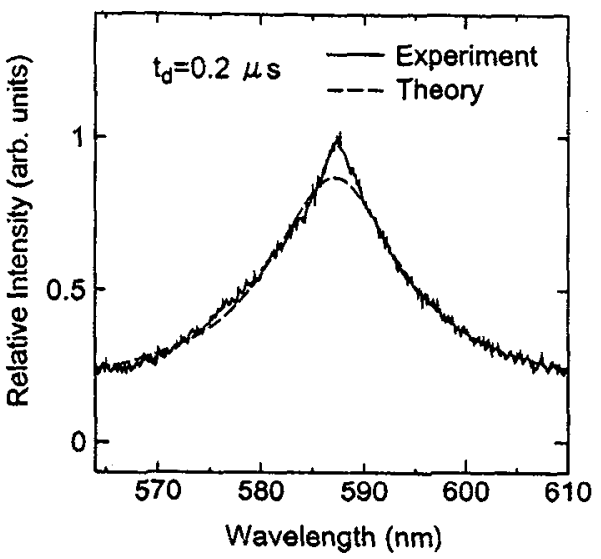

a)

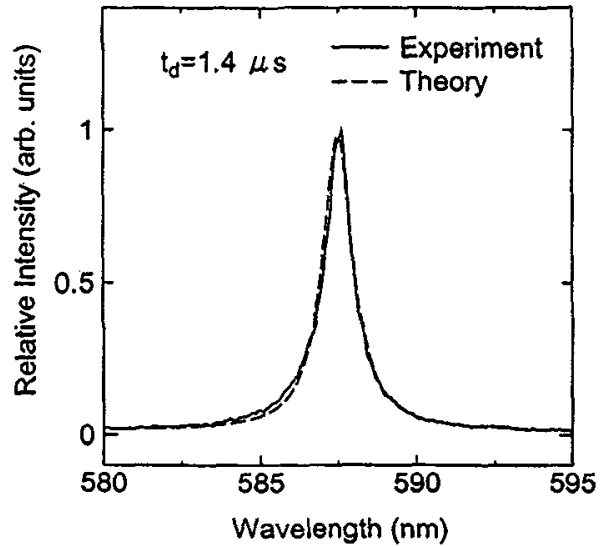

b)

Figure 3: The comparison between theoretically calculated Stark profiles and the measured spectral line shapes of spectral line $587.6 \mathrm{~nm}$ at delay time a) $0.2 \mu \mathrm{s}$ and b) $1.4 \mu \mathrm{s}$ in $1.62 \mathrm{~K}, 5.8$ Torr superfluid liquid helium.

Secondly, the plasma densities deduced from a few different isolated lines by fitting the line profiles exhibit consistency within an error limit under the assumption that the mechanism of Stark broadening dominates the spectral line profiles. The results after Debye shielding correction [9] of spectral lines $388.9 \mathrm{~nm}, 587.6 \mathrm{~nm}, 667.8 \mathrm{~nm}$ and $706.5 \mathrm{~nm}$ in case (1) are given in Table 1 . Some of the data have large deviations from others. For example, the density obtained from line $667.8 \mathrm{~nm}$ at the delay time $t_{d}=$ $1.0 \mu \mathrm{s}$ is very low. However, these inconsistencies are not worse than those in gas cases. Taking into account the experimental errors, e.g., time jitter $(\sim 0.1 \mu \mathrm{s})$ of $\mathrm{MCP}$ delay in our single shot measurements and the accuracy of numerical calculation according to Refs. 8-10, errors in density determination from He neutral atomic lines can be estimated to be as large as $35 \%$.

Table I: Plasma densities obtained by fitting different emission spectral lines for $1.62 \mathrm{~K}, 5.8$ Torr, liquid helium.

\begin{tabular}{cccccc}
\hline Spectral line (nm) & $\begin{array}{c}3 p^{3} \mathrm{P}-2 s^{3} \mathrm{~S} \\
388.9\end{array}$ & $\begin{array}{c}3 d^{3} \mathrm{D}-2 p^{3} \mathrm{P} \\
587.6\end{array}$ & $\begin{array}{c}3 d^{1} \mathrm{D}-2 p^{1} \mathrm{P} \\
667.8\end{array}$ & $\begin{array}{c}3 s^{3} \mathrm{~S}-2 \mathrm{p}^{3} \mathrm{P} \\
706.5\end{array}$ & Average \\
\hline $\begin{array}{c}\text { Plasma density }\left(10^{17} \mathrm{~cm}^{-3}\right) \\
\text { Delay time } \\
t_{d}(\mu \mathrm{s})\end{array}$ & \multicolumn{7}{c}{} \\
\hline 0.2 & -19 & 44 & - & - & 44 \\
0.6 & 24 & 18 & 18 & 20 \\
1.0 & 7.3 & 8.9 & 5.7 & 9.0 & 7.7 \\
1.4 & 3.4 & 3.2 & 2.8 & 2.9 & 3.1 \\
1.8 & 1.9 & 2.2 & 1.9 & 2.1 & 2.0 \\
2.2 & 1.5 & 1.7 & 1.4 & - & 1.5 \\
2.6 & 1.2 & 1.1 & 0.83 & - & 1.0 \\
3.0 & 1.0 & 1.0 & - & - & 1.0 \\
3.4 & 0.91 & 0.88 & - & - & 0.90 \\
3.8 & 0.81 & 0.75 & - & - & 0.78 \\
\hline
\end{tabular}

"_._" The experimental spectral line profile is too difficult to obtain because of strong continuum background or poor signal-tonoise ratio. 
Finally, the average plasma densities deduced from spectral line shifts and those obtained from the line widths show consistency within the error limit under the assumption that the mechanism of Stark broadening and shift dominates the spectral line profiles. When there exist a large number of free charged particles near the He atom emitters, the spectral lines will not only be broadened, but also shifted [8-10]: Our experimental results show such shifts, just as was expected theoretically. Despite the large error inherent in line shifts theoretically and experimentally [8-9], the plasma densities can roughly be deduced from the fitting values of the shifts. The average densities in the cases (1) obtained from shifts and widths of $388.9 \mathrm{~nm}, 587.6 \mathrm{~nm}, 667.8 \mathrm{~nm}$ and $706.5 \mathrm{~nm}$ are depicted in Figure 4.

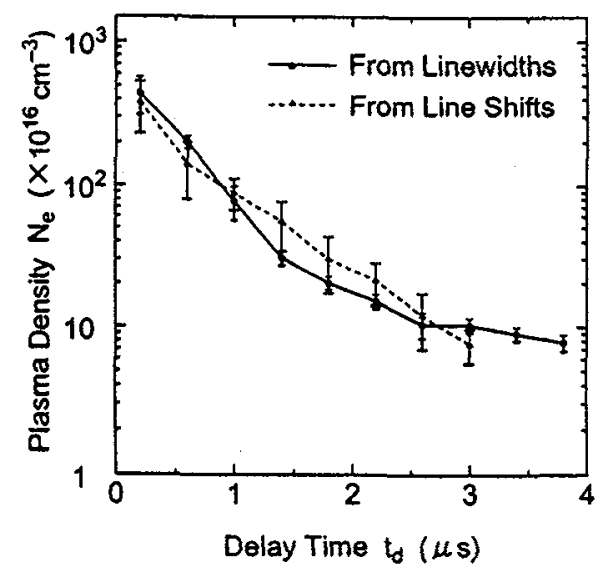

Figure 4: Comparison between average plasma densities obtained from shifts and profiles of spectral lines $388.9 \mathrm{~nm}, 587.6 \mathrm{~nm}$, $667.8 \mathrm{~nm}$ and $706.5 \mathrm{~nm}$ in $1.62 \mathrm{~K}, 5.8$ Torr superfluid liquid helium.

\subsubsection{Determination of plasma densities}

Plasma density is one of the important parameters that are used to describe a plasma environment. The value is also very important for establishing the equilibrium in the plasma. One of the advantages of measuring the density through Stark broadening is that the density can be calculated with good accuracy, even when the electron temperature $T_{e}$ is not accurately known because Stark broadening is insensitive to $T_{e}$.

In Figure 5, the average densities after Debye shielding correction, determined from line profiles of neutral lines $388.9 \mathrm{~nm}, 587.6 \mathrm{~nm}, 667.8 \mathrm{~nm}$ and $706.5 \mathrm{~nm}$, are plotted for the cases (1)-(3) as functions of the delay time $t_{d}$. The plasma densities are $4.4 \times 10^{18} \mathrm{~cm}^{-3}, 3.6 \times 10^{18} \mathrm{~cm}^{-3}$ and $1.3 \times 10^{18} \mathrm{~cm}^{-3}$ at the earliest stage of $t_{d}=0.2 \mu \mathrm{s}$ in the cases (1), (2) and (3), respectively. They are $8 \times 10^{16} \mathrm{~cm}^{-3}, 6 \times 10^{16} \mathrm{~cm}^{-3}$ and $6 \times 10^{16} \mathrm{~cm}^{-3}$, respectively, at $t_{d}=3.8 \mu \mathrm{s}$. Such plasma densities, on the order of $10^{18} \mathrm{~cm}^{-3}$, are much higher than the values, $1.4 \times 10^{17} \mathrm{~cm}^{-3}$ and $1.1 \times 10^{17} \mathrm{~cm}^{-3}$, produced, respectively, in the cases (4) and (5), at $t_{d}=0.2 \mu \mathrm{s}$. Here, it should be mentioned that, at $t_{d}=0.2 \mu \mathrm{s}$, the measured line profile may be influenced by the remains of the discharge electric field strength. However, this effect is estimated to be minor, because much lower densities are acquired under the same discharge conditions in the cases (4) and (5), and the plasma formed may also shield itself from this effect. After $3 \mu \mathrm{s}$, the electron densities in the cases (2) and (3) converge to about $6 \times 10^{16} \mathrm{~cm}^{-3}$. For the case (1), however, they remain above $7 \times 10^{16} \mathrm{~cm}^{-3}$ for a long time. Subsequent decreased emission intensities result in poor signal-to-noise ratios, and there exists a limitation in the instrumental spectral resolution. Accordingly, this phenomenon requires further investigation. 


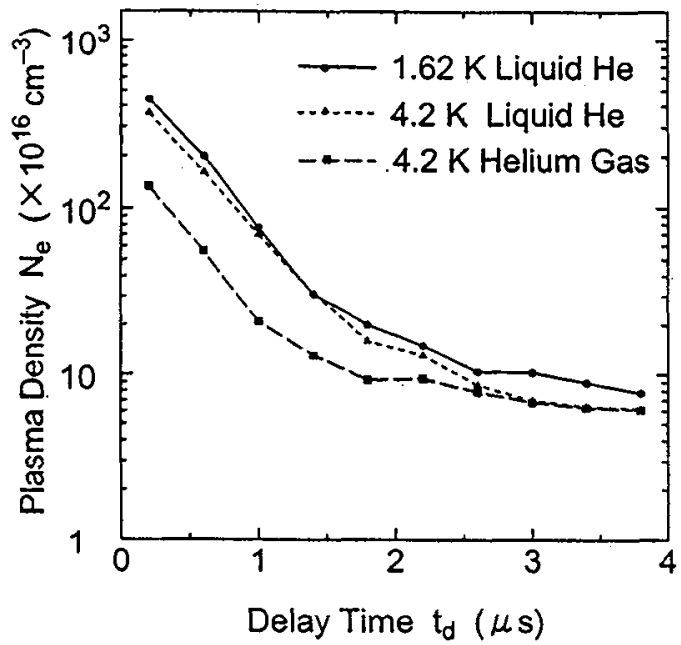

Figure 5: Plasma densities as functions of delay time $t_{d}$ in cases (1) $1.62 \mathrm{~K}, 5.8$ Torr superfluid liquid helium (He), (2) $4.2 \mathrm{~K}, 1$ atm normal fluid liquid $\mathrm{He}$ and (3) $1 \mathrm{~atm} \mathrm{He}$ gas near $4.2 \mathrm{~K}$.

In addition, in terms of the plasma densities obtained here and electron temperatures, which will be discussed in the next section, the densities of neutral He atoms at the breakdown region can be estimated by Saha equation. It turns out that the density of neutral ground-state He atoms at delay time $t_{d}=0.2 \mu \mathrm{s}$ is on the order of $10^{16} \mathrm{~cm}^{-3}$, while typical plasma density at this moment is $10^{18} \mathrm{~cm}^{-3}$ in the cases (1)-(3). This fact provides another evidence for dominant mechanism of Stark broadening.

\subsection{Determination of Electron Temperatures}

\subsubsection{Measurement of Electron Temperatures}

The observed underlying continuum light in our measurements is considered mainly to arise from recombination radiation and bremsstrahlung due to atomic processes. By using the observed continuum, the electron temperatures can be deduced by relative line-to-continuum intensities described by Griem in Ref. 8. The procedure of this method is to calculate the ratio of the measured total spectral line intensity to the continuum intensity in a certain wavelength band, e.g. $10 \mathrm{~nm}$, centered at the line. This result is then compared with the calculated one [8]. The ratio is a function of electron temperature and is independent of the plasma density. Comparing with other spectroscopic methods for electron temperature determination, this method is normally restricted to simple pure gases such as hydrogen and He, because continuum intensities can be precisely calculated. However, it permits rather precise temperature determinations in ielatively low temperatures $\left(15,000 \mathrm{~K}<T_{e}<30,000 \mathrm{~K}\right)$. As in most of other spectroscopic methods, this method is also only applied to local thermodynamic equilibrium (LTE) plasmas. The validity of LTE under our conditions will be discussed in the next subsection.

Figure 6 shows the measured $T_{e}$ as functions of delay time $t_{d}$ in the cases (1)-(3). It is found that, at delay time $t_{d}=0.6 \mu \mathrm{s}, T_{e} \cong 32,000 \mathrm{~K}, 33,000 \mathrm{~K}$ and $25,600 \mathrm{~K}$ for the cases (1)-(3), respectively. They are $19,100 \mathrm{~K}, 15,900 \mathrm{~K}$ and $17,500 \mathrm{~K}$, respectively, at $t_{d}=3.0 \mu \mathrm{s}$. The decay time of $T_{e}$ in the case (1) has some similarities to that for the case (3). In the case (2), however, the decay seems to be somewhat faster than others. The theoretical [8] and experimental uncertainty in the temperature measurements is estimated to be $20 \%$ for the delay time $t_{d}$ between $0.6 \mu \mathrm{s}$ and $3.0 \mu \mathrm{s}$ in the cases (1)_(2) 
and between $0.2 \mu \mathrm{s}$ and $3.0 \mu \mathrm{s}$ in the case (3). Before $0.6 \mu \mathrm{s}$ in LHe cases, a very strong continuum background and extremely broadened emission line profiles prevent the determination of $T_{\varphi}$. On the other hand, after $3.0 \mu \mathrm{s}$, the continuum background becomes too weak to obtain electron temperature within error $30 \%$.

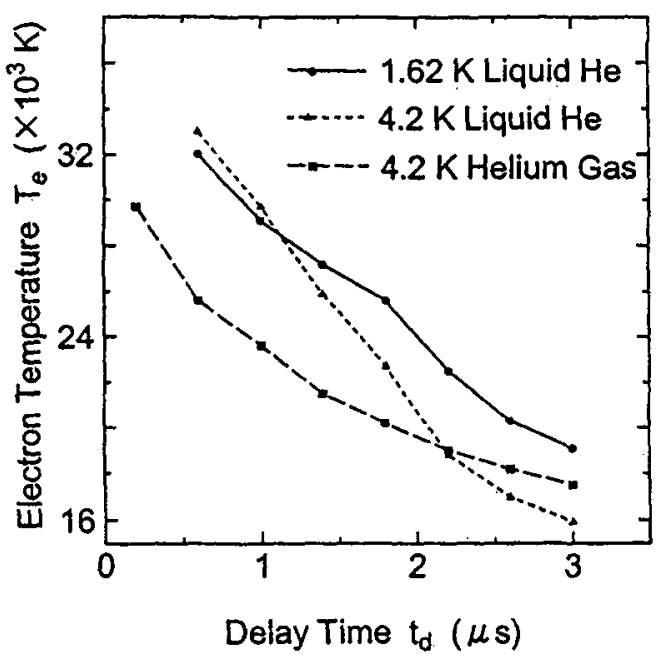

Figure 6: Electron temperatures as functions of delay time in cases (1) $1.62 \mathrm{~K}, 5.8$ Torr superfluid liquid helium (He), (2) $4.2 \mathrm{~K}$, $1 \mathrm{~atm}$ normal fluid liquid $\mathrm{He}$ and (3) I atm He gas near $4.2 \mathrm{~K}$.

\subsubsection{Equilibrium Discussions}

In order to determine electron temperature $T_{e}$ by using the method described above, it is necessary to assume that the plasma establishes a state of LTE soon after its formation. Although it is not certain that LTE exists in this transient plasma, there are some general arguments for the existence of LTE. Firstly, the lower criterion imposed on plasma density, for which the plasma will be within $10 \%$ at LTE for the excitation and ionization distributions, is $2 \times 10^{16} \mathrm{~cm}^{-3}$. From our experimental results up to $3.8 \mu$ s, the electron densities satisfy this criterion. This suggests that LTE can be attained during this time. The high plasma density and high pressure in the bubble formed by breakdown in LHe imply high collision frequencies between constituent particles. Secondly, the criterion of the time for the excitation and ionization distributions to establish equilibrium is estimated to be less than $70 \mathrm{~ns}$ in our LHe cases at $t_{d}=$ $0.2 \mu \mathrm{s}[8,11]$. The time for establishing a kinetic equilibrium between electrons, heavier neutral atoms and ions turns out to be less than $1 \mathrm{~ns}$ at $t_{d}=0.2 \mu \mathrm{s}[8,12]$. Therefore, LTE is suggested in the plasmas produced in our experiments, although it is not yet possible for us to make an experimental comparison between three temperatures, i.e., ionization, excitation and electron temperatures, directly. Nonetheless, some deviations from LTE cause no great errors in temperature determination by the method of relative line-to-continuum intensities for all neutral He lines which we have used, as long as densities are above $10^{15} \mathrm{~cm}^{-3}[8]$.

\subsection{Coulomb Coupling Coefficient $\Gamma$}

The Coulomb coupling coefficient $\Gamma$ of a plasma defined as the ratio of the average Coulomb energy to the average kinetic energy can be calculated from measured plasma densities and electron temperatures. 
According to the plasma parameters measured in the cases (1), (2) and (3), the $\Gamma$. factors of the transient plasmas are around 0.1 which is about 5 times greater than that for the case (5). Therefore, our plasmas in cryogenic environments are considered to be moderately strongly coupled plasmas.

\subsection{Analyses of Reaction Dynamics}

As one of advantages, the spectroscopic observations can provide not only plasma parameters such as density and electron temperature but also the information on reaction dynamics of the plasma.

In our experiments, the observed emissions from excited neutral atoms are very intense. These emissions were, however, completely absent in Kimura's spectra of nitrogen ion-impinged cryogenic $\mathrm{He}$ gas in this spectral range [13]. Only transitions of atomic $\mathrm{He} 706.5 \mathrm{~nm}\left(3 s^{3} \mathrm{~S}-2 p^{3} \mathrm{P}\right)$ and $728.1 \mathrm{~nm}$ $\left(3 s^{1} \mathrm{~S}-2 p^{1} \mathrm{P}\right)$ occurred in Brooks and Hunt's proton beam irradiated cryogenic He gas [14]. The failure to observe atomic lines was considered to arise from associative ionization reaction (Hornbeck-Molnar process) [15],

$$
\mathrm{He}^{*}(\mathrm{n} \geq 3)+\mathrm{He} \rightarrow \mathrm{He}_{2}^{+}+e .
$$

In our case, the concentration of neutral ground-state He atoms in the center part of breakdown is relatively low. It is reasonable that most of excited atomic He transitions occur with significant intensities as in the case (5) at the hot core of the transient plasma.

In addition to the neutral atomic spectra, some strong spectral features are observed near $23300 \mathrm{~cm}^{-1}$ $(\sim 430 \mathrm{~nm})$ in the cases (1)-(4). Here, for the convenience of assignment, unit of frequency is used. The time-resolved spectra from $22100 \mathrm{~cm}^{-1}$ to $24400 \mathrm{~cm}^{-1}(\sim 410-450 \mathrm{~nm})$ for the cases (1) and (2) are shown in Figure 7. According to the spectral data given by Orth and Ginter [16], they consist primarily of spectra of $\mathrm{He}_{2}$ transitions, $P$ branch, $g^{3} \Sigma_{g}{ }^{+}(v=0)-a^{3} \Sigma_{u}{ }^{+}(v=0)$ as marked in Figure 7. A few weak peaks

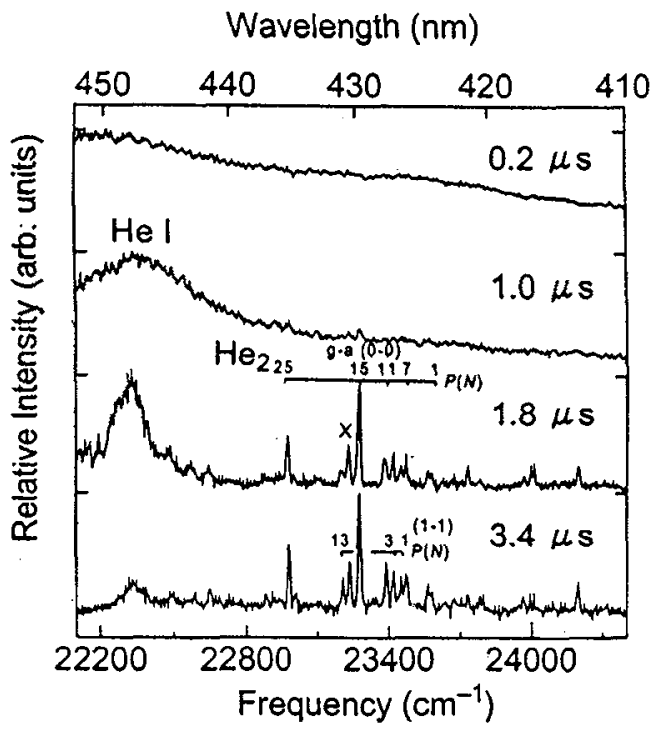

a)

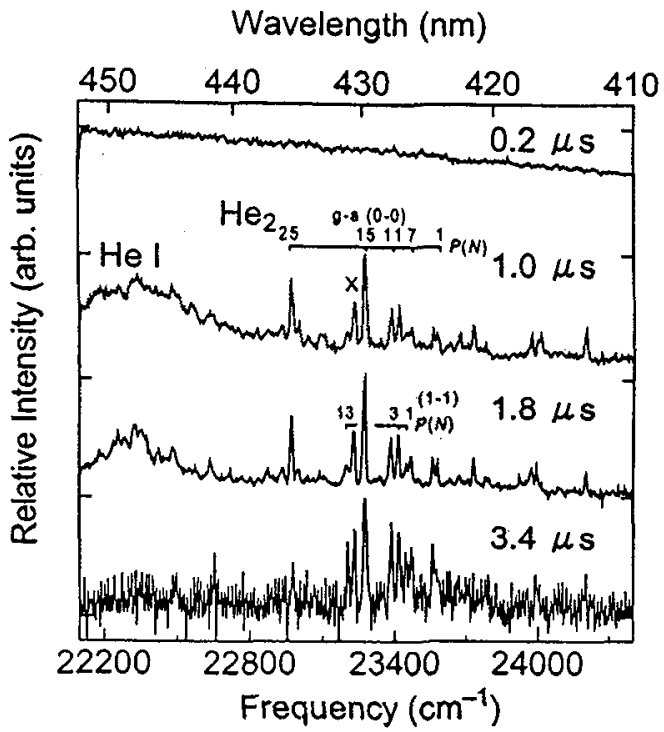

b)

Figure 7: The time-resolved emission spectra from $22100 \mathrm{~cm}^{-1}$ to $24400 \mathrm{~cm}^{-1}(\sim 410-450 \mathrm{~nm})$ at delay time $0.2 \mu \mathrm{s}, 1.0 \mu \mathrm{s}, 1.8$ $\mu \mathrm{s}$ and $3.4 \mu \mathrm{s}$ after discharges in cases a) $1.62 \mathrm{~K}, 5.8$ Torr liquid helium (LHe), and b) $4.2 \mathrm{~K}, 1 \mathrm{~atm}$ LHe. 
can be assigned to $v(1-1)$ vibrational band with $N=1,3$ and 13. No lines of the $R$ branch are identified. Here, it should be mentioned that the spectral resolution and frequency precision of our system is about 6 $\mathrm{cm}^{-1}$ at $18182 \mathrm{~cm}^{-1}(550 \mathrm{~nm})$. The assignments are accordingly limited to this precision. Some lines of the band may be ruled out because of line shift due to high pressure or line mixing. The line marked $x$ is probably caused by the transition $g-a(0-0), N=17$. However, the frequency discrepancy is as large as $11 \mathrm{~cm}^{-1}$.

The only reasonable source of excited $\mathrm{He}_{2}{ }^{*}\left(g^{3} \Sigma_{g}{ }^{+}\right)$seems to be neutralization of ionic species. No experimental observations and theoretical expectations about the formation of $\mathrm{He}_{2}{ }^{*}\left(g^{3} \Sigma_{g}{ }^{+}\right)$by other way have been reported. One of possible ionic species is $\mathrm{He}_{2}{ }^{+}$. In plasmas of high neutral density, $\mathrm{He}^{+}$ions are rapidly converted to $\mathrm{He}_{2}{ }^{+}$ions through ion conversion processes [15]:

$$
\begin{aligned}
& \mathrm{He}^{+}+2 \mathrm{He} \rightarrow \mathrm{He}_{2}^{+}+\mathrm{He}, \\
& \mathrm{He}^{+}+3 \mathrm{He} \rightarrow \mathrm{He}_{2}^{+}+2 \mathrm{He} .
\end{aligned}
$$

The associative ionization process (1) also occurs rapidly for excited atoms with levels higher than $n=2$. It is commonly believed that the $\mathrm{He}_{2}{ }^{+}$ion formed from reactions (1)-(3) are in highly excited vibrational states $[15,17]$. However, the spectra we observed in low temperature are found to be "hot" rotational distribution maximized at $N=15$ with "cool" vibrational distribution ( $\nu=0$ and 1 ) of $\mathrm{He}_{2}{ }^{*}$. The resultant molecules of the transition should be rotationally excited molecules in $a^{3} \Sigma_{u}{ }^{+}$state. As a progenitor of $\mathrm{He}_{2}{ }^{*}\left(g^{3} \Sigma_{\mathrm{g}}^{+}\right), \mathrm{He}_{2}{ }^{+}$ion probably relaxes to its ground vibrational state very fast. However, it is very difficult to picture a mechanism to explain the formation of $\mathrm{He}_{2}{ }^{*}\left(g^{3} \Sigma_{g}{ }^{+}\right)$or its progenitor, $\mathrm{He}_{2}{ }^{+}$ion, in highly rotationally excited states.

Rotational distribution of non-thermal equilibrium was observed in temperature lower than about $200 \mathrm{~K}$ by Callear and Hedges [18]. Infrared absorption spectra from metastable He molecules $\left(a^{3} \Sigma_{u}^{+}\right)$ were previously examined with proton beam irradiation by Brooks, Hunt and Tokaryk [17, 19]. It was observed that the vibrational-rotational distribution within the $a^{3} \Sigma_{u}^{+}$state was far from thermal equilibrium. There were three different vibrational-rotational groups: thermal molecules $(\nu=0, N=1)$, rotationally excited molecules $(\nu=0,9 \leq N \leq 21)$ and vibrationally excited molecules $(10 \leq \nu \leq 12, N=1)$. They suggested that the rotationally excited molecules were the products of neutralization of $\mathrm{He}_{3}{ }^{+}$ions rather than $\mathrm{He}_{2}{ }^{+}$ions, which were considered to be progenitor for the vibrational excited ones.

Evidence of the existence of $\mathrm{He}_{3}{ }^{+}$ions in low temperatures was given by Patterson [20]. It was proposed that the reaction leading to a stable $\mathrm{He}_{3}{ }^{+}$was a three-body process or a four-body reaction [17],

$$
\begin{aligned}
& \mathrm{He}_{2}^{+}+2 \mathrm{He} \rightarrow \mathrm{He}_{3}^{+}+\mathrm{He}, \\
& \mathrm{He}^{+}+3 \mathrm{He} \rightarrow \mathrm{He}_{3}^{+}+\mathrm{He} .
\end{aligned}
$$

The rate of formation of $\mathrm{He}_{3}{ }^{+}$from $\mathrm{He}_{2}{ }^{+}$was found to be faster than the formation of $\mathrm{He}_{2}{ }^{+}$through reaction (2) and (3) at $76 \mathrm{~K}$. Further studies made by other researchers indicated that $\mathrm{He}_{3}{ }^{+}$ion was, in fact, the dominant ion in $76 \mathrm{~K} \mathrm{He}$ afterglow and the neutralization of these ion species produced $\mathrm{He}_{2}{ }^{*}$ and He [21].

Obviously, this reaction model can explain our spectra very well. However, it seems to be impossible to estimate that the molecular emissions come from the hot core of the plasma. At edge layers, where the density of neutral He atoms is very high and all kinds of particles are undergoing a rapid cooling, $\mathrm{He}_{3}{ }^{+}$ions are formed quite readily. According to the intensities of the spectra presented here, $\mathrm{He}_{3}{ }^{+}$ions may be created very copiously in outer layers during and after the discharge. They lead to recombination within the time resolution of $0.4 \mu \mathrm{s}$.

In conclusions, as schematically illustrated in Fig. 8, the intense neutral atomic He spectra are considered to be from the hot core of the plasma, where the dominant positive ions are $\mathrm{He}^{+}$. The obtained plasma densities and electron temperatures in this work are spatial average values of the line-of- 
sight in this region. In contrast, the strong molecular $\mathrm{He}_{2}$ transitions are considered to occur at rapid cooling edge layers. They are suggested to be a subsequent process of neutralization of $\mathrm{He}_{3}{ }^{+}$ions. In other words, in edge region of the plasma, $\mathrm{He}_{3}{ }^{+}$are the dominant positive ions. Here, it should be noted that the so-called "hot core" and "rapid cooling edge layer" are two extreme conditions. Between these two conditions, there may exist some region where both excited atoms and molecules are emitting and the kinetics are much more complicated. The object we viewed is a very dense transient plasma with extremely large temperature and density gradients.

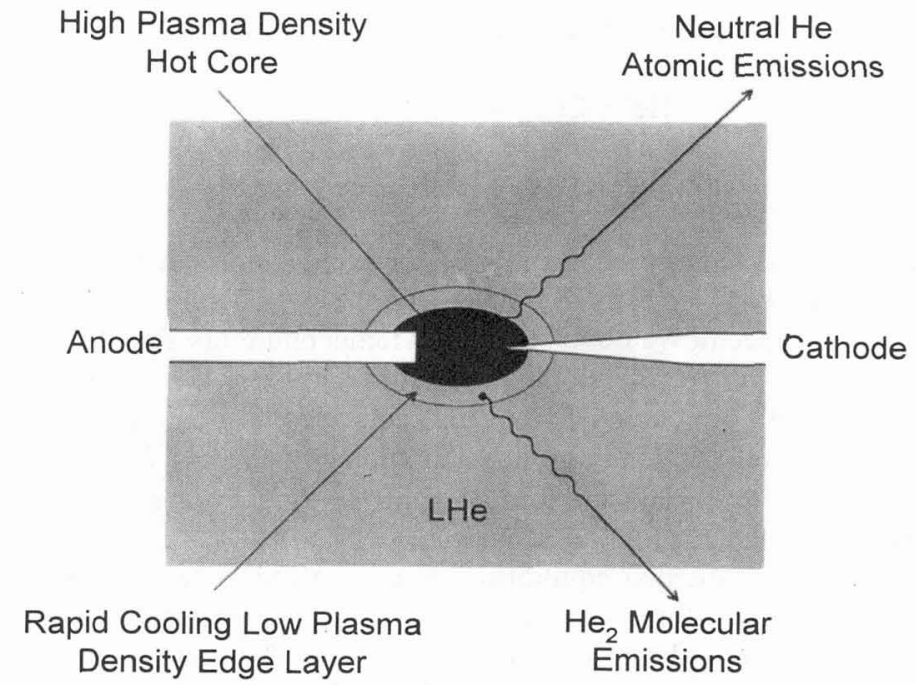

Figure 8: The schematic diagram of the discharge region.

\section{CONCLUSIONS}

The time-resolved emission spectra from high-voltage pulsed discharges in LHe below $4.2 \mathrm{~K}, 1 \mathrm{~atm}$, and cryogenic helium gas near $4.2 \mathrm{~K}, 1 \mathrm{~atm}$, have been examined in a spectral region from 380 to $720 \mathrm{~nm}$. The spectra exhibit both the well known neutral atomic lines, which are strong and broadened with underlying continua at early stages, and intense molecular $\mathrm{He}_{2}$ transitions.

Several experimental findings indicate that the broadening of neutral atomic lines in $\mathrm{LHe}$ cases is dominated by Stark broadening. The spectral profiles are therefore used to deduce the plasma densities, which are on the order of $10^{18} \mathrm{~cm}^{-3}$ at $0.2 \mu$ s after breakdown. The electron temperatures are measured from the ratios of total spectral line intensities to continuum intensities. It turns out to be about 35,000 $\mathrm{K}$ at $0.2 \mu \mathrm{s}$. The gaseous plasma state with relatively high density and low electron temperature is obtained in cryogenic environments. According to the plasma parameters obtained here, the Coulomb coupling coefficient $\Gamma$ are around 0.1 which is about 5 times greater than that in the room temperature He gas case. Therefore, the plasmas are considered to be moderately strongly coupled plasmas.

However, the obtained plasma parameters are limited to the region of the plasma hot core, where the emissions of neutral excited atoms are substantial. At rapid cooling outer layers, $\mathrm{He}_{3}{ }^{+}$ions are proposed to be dominant positive ions. The strong molecular $\mathrm{He}_{2}$ transitions are estimated to be a subsequent process of neutralization of $\mathrm{He}_{3}{ }^{+}$ions.

The present work makes the first investigation on a transient plasma produced by pulsed discharge in LHe environment. The present results would be helpful to understand the physics of very dense helium plasmas. 


\section{Acknowledgements}

The authors would like to acknowledge profoundly Professor H. R. Griem, the University of Maryland, for his interest and encouragement to present work.

\section{References}

[1] Hooper C. F., Jr., Mancini R. C., Haynes D. A., Jr. and Garber D. T., Elementary processes in dense plasmas, eds. Ichimaru. S and Ogata. S (Addison-Wesley, Tokyo, 1995) pp. 403-414.

[2] Azechi H. et al., Laser \& Particle Beams 9 (1991) 193-207.

[3] Fortov V. E., Bushman A.V., Filimonov A. S., Kvitov S. V., Kulish M. I., Lebedev M. E., Polischuk A. Ya. and Ternovoi V. Ya., Shock compression of condensed matters (Elsevier Science Publishers, New York, 1992) pp. 745-748.

[4] Mostovych A. N. and DeSilva A. W., Phys. Rev. A 34 (1986) 3238-3252.

[5] Fujisaki A., Sano K., Kinoshita T., Takahashi Y. and Yabusaki T., Phys. Rev. Lett. 71 (1993) 10391042.

[6] Cremers D. A., Radziemski L. J. and Loree T. R., Appl. Spectrosc. 38 (1984) 721-729.

[7] Minami K., Watanabe S., Qin W., Tomimoto F., Kuwabara S., Ruhul Amin Md., Kato K., and Satow T., Jpn. J. Appl. Phys. 34 (1995) 271-276.

[8] Griem H. R., Plasma spectroscopy (McGraw-Hill, New York, 1964) pp. 63-455.

[9] Griem H. R., Spectra line broadening by plasmas (Academic Press, New York and London, 1974) pp. 1-320.

[10] Griem H. R., Baranger M., Kolb A. C., and Oertel G., Phys. Rev. 125 (1962) 177-195.

[11] Griem H. R., Phys. Rev. 131 (1963) 1170-1176.

[12] Lochte-Holtgreven W., Plasma diagnostics, ed. Lochte-Holtgreven W. (Wiley, New York, 1968) pp.180-182.

[13] Kimura K., J. Chem. Phys. 84 (1986) 2002-2009.

[14] Brooks R. L. and Hunt J. L., J. Chem. Phys. 88 (1988) 7267-7272.

[15] Deloche R., Monchicourt P., Cheret M. and Lambert F., Phys. Rev. A 13 (1976) 1140-1176.

[16] Orth F. B. and Ginter M. L., J.Mol. Spectrosc. 61 (1976) 282-288.

[17] Tokaryk D. W., Brooks R. L. and Hunt J. L., Phys. Rev. A 48 (1993) 364-381.

[18] Callear A. B. and Hedges R. E. M., Trans. Faraday Soc. 66 (1970) 2921-2935.

[19] Brooks R. L. and Hunt J. L. and Tokaryk D. W., J. Chem. Phys. 91 (1989) 7408-7414.

[20] Patterson P. L., J. Chem. Phys. 48 (1968) 3625-3631.

[21] Gerber R. A. and Gusinow M. A., Phys. Rev. A 4 (1971) 2027-2033. 